\title{
Preservative-free versus preserved latanoprost eye drops for reducing intraocular pressure: a non-inferiority phase III randomized, multi-center, single-blind, parallel-group controlled trial
}

\author{
Panos Theodosiadis ${ }^{1}$, Anastasios Konstas ${ }^{2}$, Ioannis Halkiadakis ${ }^{3}$, Vasiliki Dimera ${ }^{4}$, Dimitrios Koufakis ${ }^{5}$, Constantinos D \\ Georgakopoulos ${ }^{6}$, Evgenia Kanonidou ${ }^{7}$, Elias Zintzaras ${ }^{8}$, Konstantina Soulele ${ }^{9}$, Antonios Margaritis ${ }^{9}$, Lida Kalantzi ${ }^{9}$ iD \\ ${ }^{1}$ Attikon University Hospital, Athens, Greece \\ ${ }^{2}$ General University Hospital of Thessaloniki AHEPA, Thessaloniki, Greece \\ ${ }^{3}$ Ophthalmiatreio Athens, Athens, Greece \\ ${ }^{4}$ General Hospital of Larissa, Larissa, Thessaly, Greece \\ ${ }^{5}$ IASO Thessalias, Larissa, Greece \\ ${ }^{6}$ General University Hospital of Patra, Patra, Greece \\ ${ }^{7}$ General Hospital of Thessaloniki Ippokrateio, Thessaloniki, Greece \\ ${ }^{8} \mathrm{BECRO}$, Athens, Greece \\ ${ }_{9}^{9}$ Pharmathen S.A., Attica, Greece
}

\begin{abstract}
Background: The aim of this study was to test the non-inferiority of preservative-free (PF) latanoprost $50 \mu \mathrm{g} / \mathrm{mL}$ multi-dose ophthalmic solution versus the marketed benzalkonium chloride (BAK)-preserved latanoprost $50 \mu \mathrm{g} / \mathrm{mL}$ ophthalmic solution in patients with open-angle glaucoma and patients with ocular hypertension.

Methods: This was a prospective, national, randomized, multi-center, observer-blind, parallel-group controlled clinical trial. Patients were randomized to receive either PF or BAK-preserved latanoprost once daily for 12 weeks. The primary endpoint was the change in intraocular pressure (IOP) at 8:00 AM in the affected eye between the end of the treatment (week 12) and the baseline (week 0). Secondary measurements were taken at weeks 2 and 6 , with IOP being recorded at 8:00 AM, 12:00 PM, and 4:00 PM.

Results: A total of 158 patients were included in the per protocol (PP) population (77 in the PF latanoprost treatment arm and 81 patients in the BAK-preserved latanoprost treatment arm). PF latanoprost was non-inferior to BAK-preserved latanoprost in reducing IOP at 8:00 AM in the study eye from the baseline (week 0 ) to the end of the treatment (week 12). The point estimate of the between-treatment difference was $0.1 \mathrm{mmHg}(95 \%$ confidence interval: $-0.646,0.847)$. Mean between-group differences in IOP reduction from the baseline to each of the secondary measurements were also similar between the two treatment arms. The two treatments were well tolerated and had comparable adverse event profiles.

Conclusions: PF latanoprost was non-inferior to BAK-preserved latanoprost in reducing IOP in patients with open-angle glaucoma or ocular hypertension. Both treatments were well tolerated.
\end{abstract}

\section{KEY WORDS}

glaucoma, intraocular pressure, benzalkonium chloride, latanoprost, preservative-free, eye drop, randomized controlled trial, preservative-free

Copyright (C) 2020, Author(s). This is an open-access article distributed under the terms of the Creative Commons Attribution-Non Commercial 4.0 International License (http://creativecommons.org/licenses/by-nc/4.0/) which permits copy and redistribute the material just in noncommercial usages, provided the original work is properly cited.

Correspondence to: Dr. Lida Kalantzi PhD, Head of Scientific Affairs, Pharmathen S.A., 44, Kifissias Ave., 15125 Marousi Attica, Greece, E-mail: lkalantzi@pharmathen.com

How to cite this article: Theodosiadis P, Konstas A.G, Halkiadakis I, Dimera V, Koufakis D, Georgakopoulos CD, Kanonidou E, Zintzaras E, Soulele K, Margaritis A, Kalantzi L, Preservative-free versus preserved latanoprost eye drops for reducing intraocular pressure: a non-inferiority phase III randomized, multi-center, single-blind, parallel-group controlled trial. Med Hypothesis Discov Innov Ophthalmol. 2020 Winter; 9(4): 273-283. DOI: https://doi.org/10.51329/mehdiophthal1413 


\section{INTRODUCTION}

Latanoprost is a prostaglandin analog that is widely used in the treatment of high intraocular pressure (IOP) in patients with open-angle glaucoma and patients with ocular hypertension [1]. Latanoprost is actually a prodrug: upon instillation onto the cornea, latanoprost is rapidly hydrolyzed by topical esterases in the cornea to provide the pharmacologically active latanoprost acid [2].

One way latanoprost might reduce IOP is an increase in aqueous humor outflow. This pharmacodynamic effect is mediated by two main mechanisms. The first includes the enhancement of the pressure-sensitive trabecular outflow pathway, while the second is mediated through an increase in pressure-insensitive uveoscleral outflow $[3,4]$. Following a single dose of latanoprost $0.005 \%$ ophthalmic solution, IOP reduction is maximal at $8-12$ hours postinstillation, with IOP remaining below the pretreatment levels for at least 24 hours. Thus, latanoprost administered once daily in the evening exerts a sustained IOP reduction, with the hypotensive effect being the greatest during the day $[2,5]$.

Compared with other prostaglandin analogs, ophthalmic solutions of latanoprost exhibit higher ocular tolerability with fewer adverse events (AEs), indicating a more favorable efficacy and safety profile [6]. Since latanoprost eye drops usually contain a preservative, the impact of latanoprost on the AE profile of the formulation remains controversial [7]. In fact, preservative agents are generally considered to contribute to decreased ocular surface tolerability. Especially in patients receiving chronic treatments or suffering from underlying ocular surface diseases, topical AEs of preserved antiglaucoma medications should be carefully considered, as they may significantly influence patients' compliance and quality of life [6].

Latanoprost is marketed as a $0.005 \%$ ophthalmic solution containing $0.02 \%$ preservative benzalkonium chloride (BAK) [6]. Efficacy and safety results from previous studies have suggested a similar efficacy and similar or better local tolerance of preservative-free (PF) latanoprost ophthalmic solutions compared with the marketed BAKpreserved formulation, with less conjunctival hyperemia and less subjective symptoms upon instillation (such as burning, stinging, and pruritus) [6, 8-10].

Based on these data, the industry responded positively to addressing the needs of patients with sensitivity or allergy to BAK, with a number of single-dose PF antiglaucoma formulations approved during the last years [11, 12]. However, the use of single-dose packages can have a higher cost and may also be proven problematic in a subset of patients, such as in the elderly with decreased manual dexterity, which increases the potential for microbial contamination $[13,14]$.

To accommodate this unmet patient need, a new, multidose, PF latanoprost $50 \mu \mathrm{g} / \mathrm{mL}$ eye drops solution (Pharmathen S.A., Athens, Greece) has been developed, which is user-friendly and, at the same time, capable of maintaining a high product quality. The product is packaged in a novel multi-dose container with the Aero Pump 3K technology, which offers a three-part contamination protection: a specially designed filter that protects the product from microbiological contamination by using the enclosed air necessary for volume equalization, a silver spiral on the upper fraction to hinder bacterial growth, and a special valve system that stops backflow.

Therefore, the aim of this study was to assess the noninferiority of this new PF formulation versus the BAKpreserved product (Xalatan) in reducing IOP in patients with open-angle glaucoma and patients with ocular hypertension over a 12-week treatment period.

\section{METHODS}

\section{Study design and participants}

This was a national, randomized, multi-center, observerblind, parallel-group controlled phase III clinical trial comparing PF latanoprost $50 \mu \mathrm{g} / \mathrm{mL}$ multi-dose ophthalmic solution (Pharmathen S.A., Athens, Greece) with BAK-preserved latanoprost $50 \mu \mathrm{g} / \mathrm{mL}$ (Xalatan; Pfizer Limited, Kent, UK). Due to differences in the medication packaging (PF latanoprost has a special container closure system that uses Aero Pump 3K technology), the investigator measuring the IOP was masked to the study medication. The study was conducted at seven clinical sites in Greece (General University Hospital of Athens Attikon, General University Hospital of Thessaloniki AHEPA, General Hospital of Larissa, Ophthalmiatreio Athens, IASO Thessalias, General University Hospital of Patra, and General Hospital of Thessaloniki Ippokrateio) between October 10, 2017 and February 23, 2018. The study protocol was prospectively approved by the National (Hellenic) Ethics Committee (NEC) (September 15, 2017) and the National (Hellenic) Organization for Medicines (EOF) (September 26, 2017). The current study was conducted in compliance with the Declaration of Helsinki (2004) and Good Clinical Practice (GCP) guidelines. Relevant written informed consent was obtained from the patients prior to study enrollment. The study was registered in the EU Clinical Trials Register 
database with trial identification number 2017-00291029. Eligible subjects were male or female patients $(\geq 18$ years old) with unilateral or bilateral open-angle glaucoma or ocular hypertension. Following at least 4 weeks of washout of IOP-lowering medications, at baseline, patients were required to have an average IOP $\geq 22 \mathrm{mmHg}$ and $\leq 35 \mathrm{mmHg}$ in at least one eye and a best-corrected visual acuity $\geq 20 / 100$ (Snellen) corresponding to the logarithm of minimal angle of resolution (logMAR) of 0.7 . Patients were eligible for inclusion in the study if IOP was expected by the investigator to remain controlled with the new treatment without optic nerve damage or progression of visual field loss; arterial blood pressure was controllable; and no new systemic medication that may have altered the IOP (e.g., beta-blockers, calcium channel blockers, angiotensin-converting enzyme [ACE] inhibitors, and prostaglandins) had been taken in the previous 30 days. The primary exclusion criteria were a history of chronic and recurrent inflammatory ocular disease, ocular trauma, or infections; a history of anterior chamber lens, torn posterior lens capsule, or corneal abnormalities that would preclude accurate IOP reading with an applanation tonometer; clinically significant or progressive retinal disease; narrow-angle/angle-closure glaucoma; intraocular surgery within the previous 3 months; aphakia or any known risk factor for cystoid macular edema; ocular laser surgery within the previous 1 month; cup/disk ratio $>0.8$; best-corrected visual acuity $\leq 20 / 100$ (Snellen), corresponding to worse than 0.7 logMAR score; treatment with topical, ocular, nonsteroidal anti-inflammatory drugs, local or systemic corticosteroids, or oral carbonic anhydrase inhibitors, or any change in systemic medication that affects IOP within the previous 30 days; and a history of allergic hypersensitivity or poor tolerance to any study medication component. Patients who were pregnant, breast-feeding, or of childbearing potential and not protected by a highly effective contraceptive method of birth control were also excluded from the study. The sample size of the participating population was calculated to obtain the required power for the demonstration of non-inferiority of PF latanoprost eye drops (test, T) compared to BAK-preserved latanoprost (control, C) in reducing IOP from baseline. For this calculation, the onesided significance level was set to $2.5 \%$, and the power was set to $80 \%$. A non-inferiority margin of $1.5 \mathrm{mmHg}$ was used, as this tolerance criterion is usually employed and accepted in non-inferiority glaucoma studies [14-16], assuming a common standard deviation of the betweengroup difference of 2.6, and a mean difference between the treatment groups of 0.3 . With the above assumptions, a total sample size of 150 patients was initially required to be enrolled in the study, equally distributed to the respective treatment groups. Furthermore, since the primary analysis is typically based on the per protocol (PP) population, a dropout rate of approximately $15 \%$ was further assumed, leading to a total of 170 patients (i.e., 85 patients per treatment group) being included in the study.

\section{Treatment and efficacy assessments}

Eligible patients were randomized at day 0 to receive once-daily treatment with either PF latanoprost $50 \mu \mathrm{g} / \mathrm{mL}$ or BAK-preserved latanoprost $50 \mu \mathrm{g} / \mathrm{mL}$ for a total duration of 12 weeks. Patients were instructed to instill one drop in each eye at approximately in the evening and were scheduled for follow-up visits at weeks 2,6 , and 12 . As the clinical trial was observer-blind, due to differences in the medication packaging, to ensure masking, treatment allocation was carried out by personnel other than the observer. Patients were asked at each visit about their compliance with the treatment regimen, and their answers were recorded. To further assess compliance, patients were asked to return full and empty bottles of the eye drops at the end of the clinical trial and to keep a personal diary where the date and time of each instillation was recorded. Patients should bring the diary back at every scheduled visit (weeks 2, 6, and 12).

IOP was measured using a calibrated Goldmann tonometer at 8:00 AM, 12:00 PM, and 4:00 PM ( $\pm 1 \mathrm{~h})$ at baseline and at weeks 2 ( \pm 2 days), 6 ( \pm 2 days), and 12 ( \pm 4 days). Two consecutive measurements were taken in each eye; if these two measurements differed by more than $4 \mathrm{mmHg}$, a third measurement was performed. The IOP for a given eye was calculated as the mean of two or three IOP measurements. Diurnal IOP was defined as the mean IOP at all time points during a visit. In patients with bilateral disease, where one of the two eyes satisfied the eligibility criteria (study eye), the contralateral eye was treated with BAK-preserved latanoprost $50 \mu \mathrm{g} / \mathrm{mL}$, and the study eye was randomly assigned to one of the two study groups. If the criteria for evaluation were fulfilled for both eyes, the eye with the higher IOP at baseline was included.

\section{Safety assessments}

Safety evaluations included both ocular and systemic AEs, including a decrease in visual acuity as compared to baseline, changes in ocular signs based on slit-lamp biomicroscopy examination, subjective ocular findings (irritation, stinging, burning, eye dryness, and foreign body sensation), and vital signs (heart rate and blood pressure). The safety population (SP) consisted of all patients receiving at least one eye drop of PF or BAK-preserved latanoprost. Descriptive statistics were used to summarize the safety data (mean and standard deviation). Safety data were also compared, when appropriate, using the chi-square test or Fisher's exact test, 
based on the nature of the obtained data. Statistical significance was set at $P<0.05$. All analyses were performed using IBM SPSS Statistics for Windows, version 24 (IBM Corp., Armonk, NY, USA).

\section{Efficacy endpoints and statistical analyses}

The intent-to-treat (ITT) population included all randomized patients who had at least one post-baseline IOP measurement. The PP population included all randomized patients who had no major protocol violations, completed IOP measurements, completed at least 12 weeks of treatment, and did not take prohibited medication. A primary efficacy analysis was performed in the PP population. For collateral purposes, an efficacy assessment was conducted on the ITT population. Missing data were not imputed. The primary efficacy endpoint to prove noninferiority was the change in IOP at 8:00 AM in the study eye from baseline to the end of the treatment (week 12). Comparison of treatments was performed using an analysis of covariance model (ANCOVA), with treatment as the main effect and baseline IOP as a covariate. The test medication was considered to be non-inferior to the control medication if the upper $95 \%$ confidence interval $(\mathrm{Cl})$ limit for the difference $(\mathrm{T}-\mathrm{C}$ ) was $<\Delta \mathrm{NI}$, where $\Delta \mathrm{NI}=1.5 \mathrm{mmHg}$ was the defined non-inferiority criterion. This non-inferiority criterion is commonly used and accepted in non-inferiority glaucoma studies [14-16]. The center-by-treatment interaction was included as a fixed effect in the ANCOVA model for the primary endpoint. If the interaction was not significant, it was omitted from the analysis. Secondary efficacy endpoints included changes in IOP from baseline to each follow-up time point (week 12: 12:00 PM and 4:00 PM; weeks 2 and 6: 8:00 AM, 12:00 PM, and 4:00 PM). Secondary efficacy analysis was performed using ANCOVA, with treatment as the main effect and baseline IOP as a covariate. The treatment difference and two-sided $95 \% \mathrm{Cl}$ for the difference were also obtained for the secondary endpoints.

Ancillary statistical analyses in the PP population included ANCOVA to further assess the change in diurnal IOP from baseline to week 12 (with treatment as the main effect and baseline diurnal IOP as covariate). Additionally, a repeated measures general linear model was applied to investigate the trend of IOP over time, with treatment as a between-subjects factor and IOP at baseline, week 2, week 6, and week 12, or diurnal IOP as within-subjects variables. Analyses were performed for IOP measurements at 8:00 AM, 12:00 PM, and 4:00 PM and for the mean diurnal IOP.

\section{RESULTS}

\section{Patient distribution and baseline characteristics}

A total of 170 patients were randomized into the PF latanoprost group (86 patients) or the BAK-preserved latanoprost group (84 patients) (Figure 1). Of these, 158 patients (77 patients in the PF latanoprost group and 81 patients in the BAK-preserved latanoprost group) were finally included in the PP population. Twelve patients (7.1\%; 9 patients in the PF latanoprost group and 3 patients in the BAK-preserved latanoprost group) dropped out of the clinical trial, and the reasons are detailed in Figure 1.

The baseline characteristics of the patients are listed in Table 1 . The demographic and clinical characteristics were comparable between the two treatment groups at baseline. The treatment groups showed no statistically significant differences $(P>0.05)$ in terms of demographic and baseline clinical characteristics. All enrolled patients had well-controlled IOP before inclusion in the study. The difference in IOP between the test and control groups at all three baseline measurements at 8:00 AM, 12:00 PM, and 4:00 PM was not significant $(P>0.05$ for all measurements). Treatment compliance was fairly good: $98.8 \%, 100 \%$, and $100 \%$ for the test medication and $100 \%, 100 \%$, and $100 \%$ for the control medication at weeks 2,6 , and 12 , respectively.

\section{Efficacy analysis}

The evaluation of non-inferiority of PF latanoprost versus BAK-preserved latanoprost was based on the PP population, as already mentioned. Both treatment groups showed a statistically significant mean decrease from baseline in the study eye IOP at all measured time points. The recorded mean changes in IOP from baseline until the end of the study ranged from $-6.51 \mathrm{mmHg}$ to $-8.23 \mathrm{mmHg}$ for the $\mathrm{PF}$ latanoprost group and from $-6.8 \mathrm{mmHg}$ to $-8.16 \mathrm{mmHg}$ for the BAK-preserved latanoprost group (Figure 2). Treatment compliance was similar between the two treatment groups. PF latanoprost was non-inferior to BAK-preserved latanoprost for the primary efficacy endpoint, that is, the change in IOP at 8:00 AM in the study eye from baseline (week 0 ) to the end of the treatment (week 12). The point estimate of the between-treatment difference was 0.10 $\mathrm{mmHg}(95 \% \mathrm{Cl}:-0.65,0.85)$ in the PP population, with the upper limit of the $95 \% \mathrm{Cl}$ not exceeding the non-inferiority margin of $1.5 \mathrm{mmHg}$ (Table 2, Figure 3).

The center-by-treatment interaction was not significant $(P$ $=0.781)$; therefore, it was omitted from the ANCOVA model. Non-inferiority of the two treatments was further confirmed in the ITT population, with the betweentreatment difference being $0.08 \mathrm{mmHg}(95 \% \mathrm{Cl}:-0.681$, 0.836 ) and the upper limit of the $95 \% \mathrm{Cl}$ not exceeding the non-inferiority margin of $1.5 \mathrm{mmHg}$. Statistically nonsignificant differences between the two treatment arms in changes in IOP from baseline at all-time points of each visit were further obtained during evaluation of the secondary 
efficacy endpoints, which supported the primary efficacy analysis (Table 2). The mean diurnal IOP reductions from baseline to week 12 were also similar between the two treatment arms (Table 2). Ancillary analyses in the PP population performed for IOP measurements at 8:00 AM, 12:00 PM, 4:00 PM and, also, for the diurnal IOP, by means of repeated measures in ANOVA, revealed a statistically significant effect of time (baseline and weeks 2,6 , and 12) $(P<0.001)$, while the treatment effect and the interaction between treatment and time were not significant $(P>$ $0.05)$, indicating that the variability of the treatment effect was consistent over time.

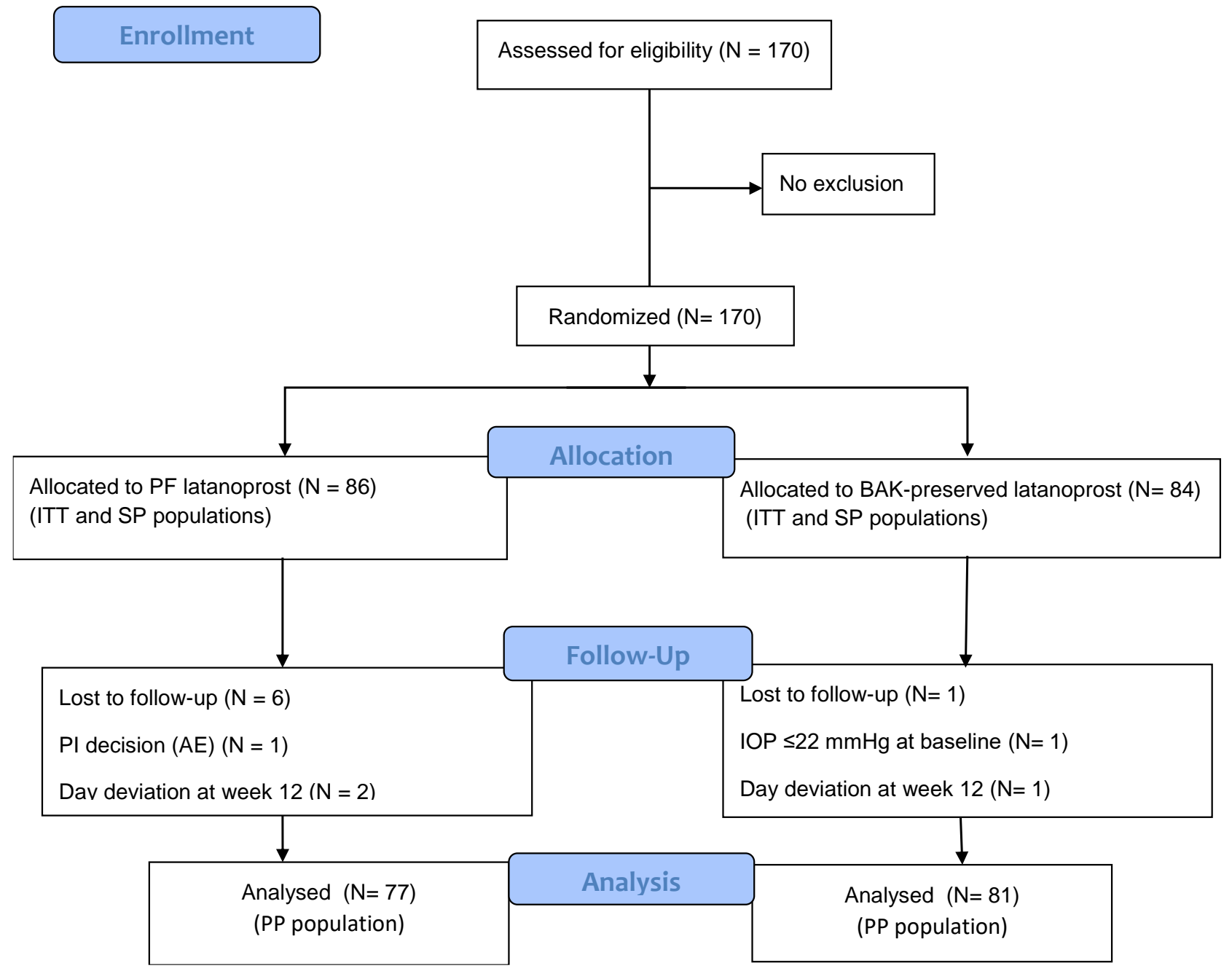

Figure 1. Patient allocation into the PF latanoprost (Pharmathen S.A.) or BAK-preserved latanoprost (Xalatan). Abbreviations: N, number; PF, preservativefree; $\mathrm{BAK}$, benzalkonium chloride; ITT, intent to treat; SP, safety population; $\mathrm{PI}$, principal investigator; $\mathrm{AE}$, adverse event; IOP, intraocular pressure; mmHg, millimeter of mercury; PP, per protocol. 
Table 1. Patient demographics and baseline characteristics of the PP population.

\begin{tabular}{|c|c|c|c|}
\hline & $\begin{array}{l}\text { PF latanoprost } \\
(\mathrm{N}=77)\end{array}$ & $\begin{array}{l}\text { BAK-preserved latanoprost } \\
(\mathrm{N}=\mathbf{8 1})\end{array}$ & $P$-value \\
\hline Age, years Mean (SD) & $70.1(9.5)$ & $69.3(9.2)$ & 0.615 \\
\hline Sex, Woman, n (\%) & $47(61.0)$ & $53(65.4)$ & 0.567 \\
\hline Caucasian, $\mathbf{n}(\%)$ & $77(100.0)$ & $81(100.0)$ & 0.999 \\
\hline Open-angle glaucoma, $\mathrm{n}(\%)$ & $55(71.4)$ & $51(63.0)$ & 0.258 \\
\hline Bilateral disease (\%) & $64(83.1)$ & $65(80.2)$ & 0.641 \\
\hline Comorbidities, n (\%) & $62(80.5)$ & $68(84.0)$ & 0.572 \\
\hline History of allergies, $n$ (\%) & $6(7.8)$ & $4(4.9)$ & 0.461 \\
\hline Concomitant medications, n (\%) & $59(76.6)$ & $67(82.7)$ & 0.341 \\
\hline History of ocular surgery, $\mathrm{n}(\%)$ & $25(32.5)$ & $29(35.8)$ & 0.659 \\
\hline History of ocular laser surgery, $n(\%)$ & $4(5.2)$ & $2(2.5)$ & 0.370 \\
\hline IOP (mmHg) at 8:00 AM [mean (SD)] & $24.6(2.3)$ & $24.2(1.8)$ & 0.281 \\
\hline IOP (mmHg) at 12:00 PM [mean (SD)] & $24.4(2.0)$ & $24.1(1.9)$ & 0.424 \\
\hline IOP (mmHg) at 4:00 PM [mean (SD)] & $24.0(2.0)$ & $23.7(1.9)$ & 0.266 \\
\hline Diurnal mean IOP (mmHg) [mean (SD)] & $24.3(1.9)$ & $24.0(1.7)$ & 0.281 \\
\hline
\end{tabular}

PP, per protocol; BAK, benzalkonium chloride; $n$, number; \%, percentage; IOP, intraocular pressure; mmHg, millimeter of mercury; PF, preservative-free; SD, standard deviation.

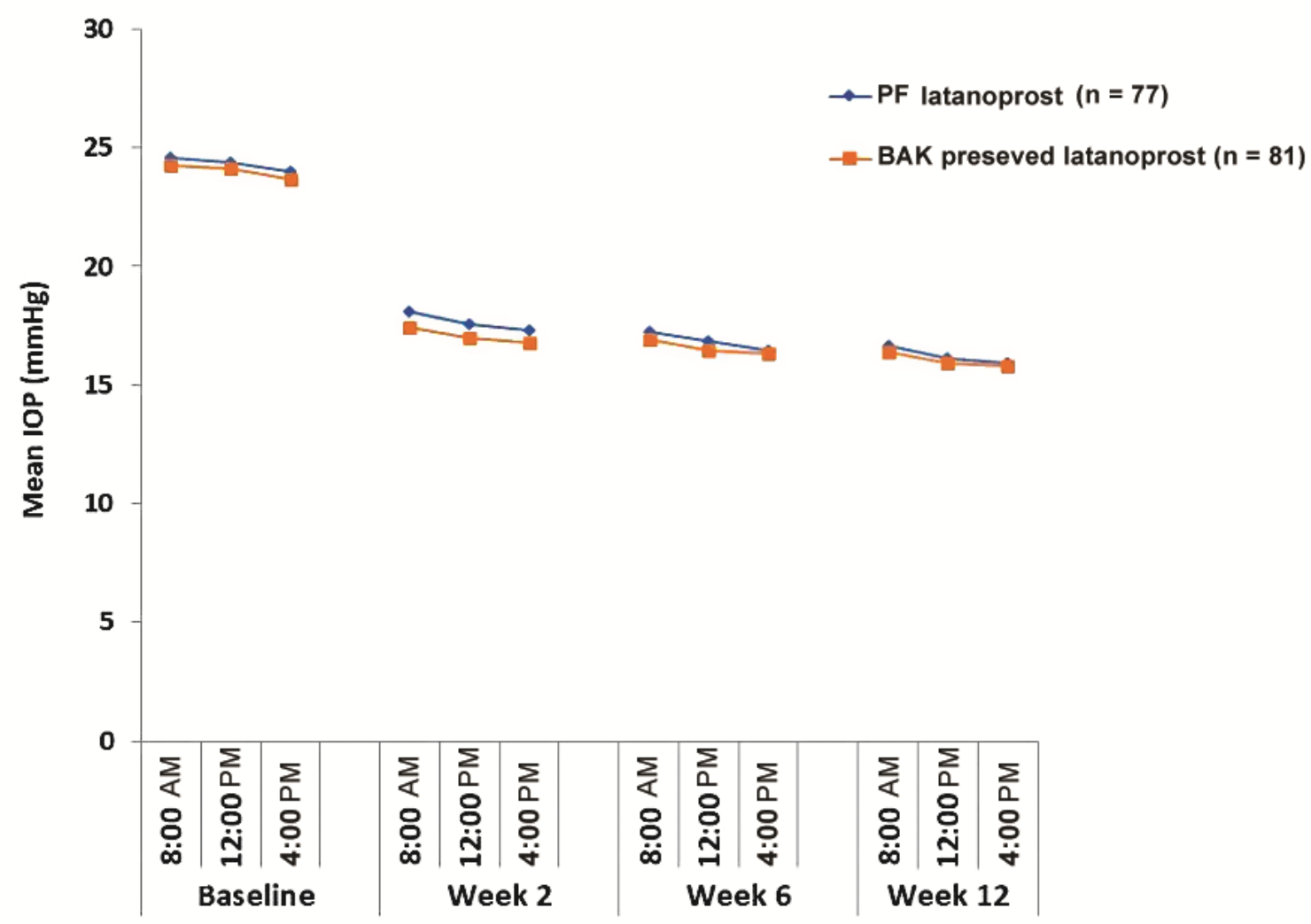

Figure 2. Mean IOP ( $\mathrm{mmHg}$ ) at each assessment time for the per protocol population. Abbreviations: PF, preservative-free; BAK, benzalkonium chloride; $\mathrm{N}$, number; IOP, intraocular pressure; $\mathrm{mmHg}$, millimeter of mercury. 


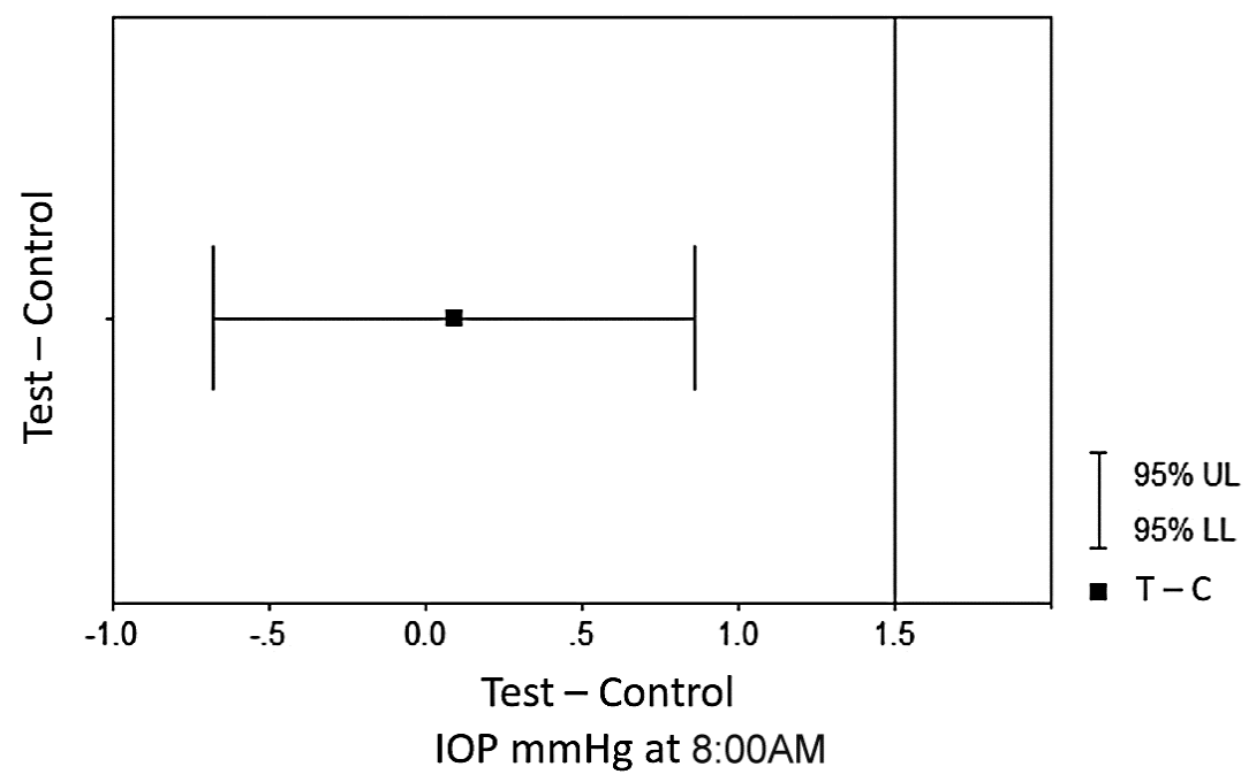

Figure 3. Forest plot showing the mean between-group difference in the change from baseline IOP. The least square mean and $95 \%$ confidence interval are presented. C, control; IOP, intraocular pressure; $\mathrm{mmHg}$, millimeter of mercury; LL, lower limit; T, test; UL, upper limit. The medication used in the test group: PF latanoprost (Pharmathen S.A.), and the medication used in the control group: BAK-preserved latanoprost (Xalatan).

Table 2. IOP change and $95 \%$ Cls for the difference in IOP from baseline to the pre-specified time points for PF and BAK-preserved latanoprost in the PP population.

\begin{tabular}{|c|c|c|c|c|c|c|c|}
\hline \multicolumn{8}{|c|}{ Time $\quad$ Mean \pm SD IOP $(\mathrm{mmHg})$} \\
\hline \multicolumn{8}{|c|}{ Primary efficacy endpoint } \\
\hline & \multicolumn{2}{|c|}{ Baseline } & \multicolumn{5}{|c|}{ Week 12} \\
\hline & PF & BAK & PF & BAK & Difference $^{a}$ & $95 \% \mathrm{Cl}^{\mathrm{b}}$ & $P$-value \\
\hline 8:00 AM & $24.59 \pm 2.30$ & $24.24 \pm 1.77$ & $16.65 \pm 2.66$ & $16.36 \pm 2.55$ & 0.10 & $(-0.65,0.85)$ & 0.791 \\
\hline \multicolumn{8}{|c|}{ Secondary efficacy endpoints } \\
\hline & \multicolumn{2}{|c|}{ Baseline } & \multicolumn{5}{|c|}{ Week 2} \\
\hline & PF & BAK & PF & BAK & Difference & $95 \% \mathrm{Cl}$ & $P$-value \\
\hline 8:00 AM & $24.59 \pm 2.30$ & $24.24 \pm 1.77$ & $18.08 \pm 2.64$ & $17.43 \pm 2.79$ & 0.43 & $(-0.33,1.19)$ & 0.267 \\
\hline 12:00 PM & $24.37 \pm 2.00$ & $24.12 \pm 1.90$ & $17.54 \pm 2.50$ & $7.01 \pm 2.78$ & 0.39 & $(-0.36,1.17)$ & 0.313 \\
\hline 4:00 PM & $24.00 \pm 1.98$ & $23.65 \pm 1.92$ & $17.30 \pm 2.41$ & $16.76 \pm 2.63$ & 0.39 & $(-0.36,1.14)$ & 0.309 \\
\hline \multirow[t]{3}{*}{ Diurnal } & $24.32 \pm 1.94$ & $24.00 \pm 1.72$ & $17.64 \pm 2.42$ & $17.07 \pm 2.66$ & - & - & \\
\hline & \multicolumn{2}{|c|}{ Baseline } & \multicolumn{4}{|c|}{ Week 6} & \\
\hline & PF & BAK & PF & BAK & Difference & $95 \% \mathrm{Cl}$ & $P$-value \\
\hline 8:00 AM & $24.59 \pm 2.30$ & $24.24 \pm 1.77$ & $17.24 \pm 2.42$ & $16.89 \pm 2.42$ & 0.17 & $(-0.52,0.86)$ & 0.626 \\
\hline 12:00 PM & $24.37 \pm 2.00$ & $24.12 \pm 1.90$ & $16.88 \pm 2.33$ & $16.43 \pm 2.48$ & 0.35 & $(-0.37,1.07)$ & 0.338 \\
\hline 4:00 PM & $24.00 \pm 1.98$ & $23.65 \pm 1.92$ & $16.47 \pm 2.35$ & $16.33 \pm 2.48$ & 0.01 & $(-0.72,0.74)$ & 0.982 \\
\hline \multirow[t]{3}{*}{ Diurnal } & $24.32 \pm 1.94$ & $24.00 \pm 1.72$ & $16.86 \pm 2.27$ & $16.55 \pm 2.37$ & - & - & \\
\hline & \multicolumn{2}{|c|}{ Baseline } & \multicolumn{4}{|c|}{ Week 12} & \\
\hline & PF & BAK & PF & BAK & Difference & $95 \% \mathrm{Cl}$ & $P$-value \\
\hline 12:00 PM & $24.37 \pm 2.00$ & $24.12 \pm 1.90$ & $16.13 \pm 2.42$ & $15.95 \pm 2.49$ & 0.10 & $(-0.65,0.84)$ & 0.800 \\
\hline 4:00 PM & $24.00 \pm 1.98$ & $23.65 \pm 1.92$ & $15.91 \pm 2.37$ & $15.83 \pm 2.49$ & -0.02 & $(-0.77,0.74)$ & 0.967 \\
\hline Diurnal & $24.32 \pm 1.94$ & $24.00 \pm 1.72$ & $16.23 \pm 2.41$ & $16.05 \pm 2.43$ & 0.05 & $(-0.67,0.78)$ & 0.330 \\
\hline
\end{tabular}

aPF minus BAK; ${ }^{b}$ for all primary and secondary endpoints, the results were non-significant $(P>0.05)$. Abbreviations: $\mathrm{PP}$, per protocol; $\mathrm{Cl}$, confidence interval; PF, preservative-free; BAK, benzalkonium chloride; IOP, intraocular pressure; $\mathrm{mmHg}$, millimeter of mercury; \%, percentage; SD, standard deviation; AM, ante meridiem or before midday; PM, post meridiem or after midday. 
Table 3. Summary of adverse events for the safety population.

\begin{tabular}{|c|c|c|}
\hline & $\begin{array}{l}\text { PF latanoprost } \\
(\mathrm{N}=\mathbf{8 6})\end{array}$ & $\begin{array}{l}\text { BAK-preserved latanoprost } \\
(\mathrm{N}=\mathbf{8 4})\end{array}$ \\
\hline Deaths, n (\%) & $0(0)$ & $0(0)$ \\
\hline Serious AEs, $\mathbf{n}(\%)$ & $0(0)$ & $0(0)$ \\
\hline Discontinuation due to AEs, $n$ (\%) & $1(1.2)$ & $0(0)$ \\
\hline Patients with $\geq 1 \mathrm{AE}, \mathrm{n}$ (\%) & $22(25.6)$ & 31 (36.9) \\
\hline Total number of AEs, $n(\%)$ & $39(45.3)$ & $45(53.6)$ \\
\hline OCULAR AEs, n (\%) & 39 (48.1) & $42(51.9)$ \\
\hline Mild ocular AEs, n (\%) & $31(79.5)$ & $28(66.7)$ \\
\hline Blepharitis, $\mathrm{n}(\%)$ & $0(0)$ & $1(2.4)$ \\
\hline Eye redness, $\mathrm{n}(\%)$ & $1(2.6)$ & $2(4.8)$ \\
\hline Eye dryness, $n$ (\%) & $3(7.7)$ & $3(7.1)$ \\
\hline Punctate keratitis, n (\%) & $0(0)$ & $1(2.4)$ \\
\hline Instillation site burning, $\mathrm{n}(\%)$ & $8(20.5)$ & $8(19.1)$ \\
\hline Itching, $\mathrm{n}(\%)$ & $0(0)$ & $2(4.8)$ \\
\hline Blurred vision, $n(\%)$ & $3(7.7)$ & $3(7.1)$ \\
\hline Eye stinging, $\mathrm{n}(\%)$ & $4(10.3)$ & $2(4.8)$ \\
\hline Foreign body sensation, $\mathbf{n}(\%)$ & $4(10.3)$ & $4(9.5)$ \\
\hline Eye irritation, $n(\%)$ & $2(5.1)$ & $2(4.8)$ \\
\hline Patient uncooperative, $n$ (\%) & $2(5.1)$ & $0(0)$ \\
\hline Moderate ocular AEs, n (\%) & $8(20.5)$ & $14(33.3)$ \\
\hline Increased IOP, n (\%) & $8(20.5)$ & $8(19.1)$ \\
\hline No IOP control ( $\geq 22 \mathrm{mmHg}$ ), $\mathrm{n}(\%)$ & $0(0)$ & $1(2.4)$ \\
\hline Conjunctival hyperemia, $\mathbf{n}(\%)$ & $4(10.3)$ & $5(11.9)$ \\
\hline Severe ocular AEs, n (\%) & $0(0)$ & $0(0)$ \\
\hline SYSTEMIC AEs, n (\%) & $0(0)$ & $3(3.6)$ \\
\hline Mild systemic AEs, $n$ (\%) & $0(0)$ & $2(66.7)$ \\
\hline Headache, n (\%) & $0(0)$ & $1(33.3)$ \\
\hline Skin erythema, $n$ (\%) & $0(0)$ & $1(33.3)$ \\
\hline Moderate systemic AEs, n (\%) & $0(0)$ & $1(33.3)$ \\
\hline Periocular skin irritation, $\mathrm{n}(\%)$ & $0(0)$ & $1(33.3)$ \\
\hline Severe systemic AEs, n (\%) & $0(0)$ & $0(0)$ \\
\hline
\end{tabular}

Abbreviations: N, number; \%, percentage; AE, adverse event; BAK, benzalkonium chloride; IOP, intraocular pressure; PF, preservative-free.

\section{Safety and tolerability}

PF latanoprost and BAK-preserved latanoprost demonstrated comparable safety profiles. No serious treatment-related AEs (including deaths) were reported. One premature discontinuation due to ocular AEs was reported (IOP increased $\geq 22 \mathrm{mmHg}$ ) in the PF latanoprost treatment arm. The SP consisted of 86 patients in the PF latanoprost arm and 84 patients in the BAK-preserved latanoprost arm. In total, 39 AEs were reported in 22 patients in the PF latanoprost group (25.6\%) and 45 AEs in 31 patients in the BAK-preserved latanoprost group (36.9\%). The vast majority of AEs were ocular and had similar intensity and frequency between the two groups (Table 3). The most common ocular AEs were instillation site burning and increased IOP ( $\geq 22 \mathrm{mmHg}$ ), followed by conjunctival hyperemia, foreign body sensation, blurred vision, eye stinging, eye dryness, eye irritation, eye redness, and blepharitis. No significant observations were noted in slit-lamp biomicroscopy findings in visual acuity change from baseline and vital signs in any of the two treatment arms. Of the three systemic AEs reported in the BAK-preserved latanoprost treatment arm, two were mild and one was of moderate intensity.

\section{DISCUSSION}

This phase III, randomized, single-blind controlled study demonstrated the non-inferiority of a novel multi-dose PF latanoprost eye drop formulation compared to the BAKpreserved formulation in terms of reduction in IOP during a 12-week treatment period. 
Both treatment groups showed a similar statistically significant mean decrease from baseline, in the study eye IOP, to the first assessment time-point (i.e., 2 weeks after treatment initiation), which was maintained for the entire duration of the study. Mean change in IOP from baseline up to 12 weeks ranged from -6.51 to $-8.23 \mathrm{mmHg}$ for the PF latanoprost group and from -6.8 to -8.16 for the BAKpreserved latanoprost group. The point estimate for the primary efficacy endpoint was $0.10 \mathrm{mmHg}$ in the PP population, with the upper limit of the range of the optimal reduction in IOP not exceeding the non-inferiority margin of $1.5 \mathrm{mmHg}$. PF latanoprost and BAK-preserved latanoprost formulations also presented a comparable safety profile, with no serious treatment-related AEs being reported.

A clear trend has been observed over the last decade in the development and clinical use of PF ophthalmic solutions. Numerous clinical studies have demonstrated that switching from preserved to PF eye drops may alleviate ocular surface symptoms while maintaining efficacy at the same level $[7,8]$. In the same vein, the substitution of preserved by PF topical antiglaucoma medications has been associated with an important increase in tear break-up time and amelioration of corneal staining and ocular surface disease index [6]. Therefore, published studies in the literature support the fact that PF prostaglandin ophthalmic solutions may present improved safety profiles concerning ocular-surface adverse events when compared to BAK-preserved solutions. BAK, the preservative used in latanoprost ophthalmic solution and the most commonly used ocular preservative, has been implicated in cases of ocular side effects, including conjunctival hyperemia. Previous studies have demonstrated that a high number of patients with glaucoma treated with topical eye drugs may have other underlying ocular surface diseases. In particular, as many as half of these patients appear to suffer from dry eye symptoms [17]. Furthermore, it has been suggested that extended exposure to BAK-preserved eye drops before filtering surgery may in some cases negatively affect surgical outcomes [18]. Based on these data, the emergence of a new generation of PF antiglaucoma medications is important, and BAK-free ophthalmic solutions should be available and used whenever required. Especially in patients suffering from pre-existing ocular surface diseases, or those with exposure to prolonged and concomitant preserved eye drop treatments, a condition that is often encountered in glaucoma patients, PF treatments could lead to better therapeutic adherence and potentially improve patients' quality of life [8].
The current study was designed to assess the noninferiority of the test PF product in terms of efficacy parameters, namely reduction in IOP, compared to the control BAK-preserved product. The two products had a similar safety profile, with a slightly improved topical ocular tolerance of the PF formulation (i.e., fewer ocular AEs with lower severity and no systemic AEs). However, due to the current study setting, no conclusive evidence can be drawn in terms of safety, apart from the actual reporting of AEs recorded during the study.

Until recently, only preserved eye drop solutions of latanoprost have been available. The presence of BAK was deemed mandatory for preventing bacterial contamination of the solution; however, new techniques and devices are now available that warrant the safe deployment of PF eye drop solutions [19]. Indeed, PF latanoprost formulations are now available, and clinical data show that these ophthalmic solutions can alleviate potential ocular surface symptoms and improve patient compliance $[20,21]$. As demonstrated by different phase II and phase III studies with the first PF latanoprost singledose unit formulation (Monoprost; Laboratoires Théa, Clermont Ferrand, France), the efficacy of PF latanoprost was somewhat better than that of preserved latanoprost $[6,20]$.

Similarly, the present study demonstrated the noninferiority of a new multi-dose PF latanoprost ophthalmic solution compared to the traditional multi-dose BAKpreserved latanoprost, in terms of IOP reduction. The percentage of IOP reduction observed from baseline to week 12 (32.3\% for PF latanoprost and $32.51 \%$ for BAKpreserved latanoprost) was within the range of optimal IOP reduction (between $-22 \%$ and $-39 \%$ ), which was also observed with other marketed PF latanoprost ophthalmic solutions $[6,8,20,21]$. Similar reductions in IOP were observed for the two formulations throughout the duration of the study, with comparable efficacy at all intermediate time points, and a similar AE profile.

A potential limitation of the current study was the selection of a single morning IOP assessment as the primary endpoint for the comparison of the two formulations. However, this methodology is supported by several clinical arguments, including: i) the fact that in most patients with glaucoma, peak IOP generally occurs in the morning; ii) the plethora of previously published noninferiority studies following the same methodology; and iii) preclinical and clinical studies indicating that the morning time point is the most challenging time to demonstrate non-inferiority in IOP between two formulations $[9,22]$. Thus, it can be suggested that the IOP-lowering effect of PF latanoprost compared to the 
BAK-preserved formulation can be extrapolated from the single morning IOP efficacy endpoint.

Another potential limitation was the single-blinded nature of the study, as lack of treatment masking could potentially influence patients' feelings regarding their treatment. A double-masked design might have been more appropriate, but this was not feasible because of the differences in the packaging systems between the two drugs; the investigational medicinal product is a PF preparation, which has a special container closure system. It should be noted, however, that the applied study procedures warranted that single masking was preserved and controlled by the study personnel, other than the principal investigator, and also by instructing study participants not to disclose information relevant to study treatment to the investigator.

Finally, a third limitation that can be considered was the rather short 12-week treatment period of the current study, which may not allow for an adequate investigation of the long-term side effects of the two latanoprost formulations. However, the current study setting was designed to allow for the assessment of the non-inferiority of the test PF product in terms of efficacy parameters (i.e., reduction in IOP) and to record the $A E$ profiles of the two drugs. In this respect, the 12 -week study period is a wellestablished treatment duration for topically applied prostaglandin analogs [6, 21].

Overall, the findings of the present study further confirmed that the IOP-lowering efficacy of latanoprost is not dependent on the presence of BAK, confirming previously reported results [6]. In fact, it has been suggested that BAK, through its detergent activity, may improve the effectiveness of topically applied drugs by enhancing their penetration into the eye and delivery into the cornea [17]. Indeed, PF prostaglandin-containing eye drops have shown decreased epithelial permeability and better maintenance of membrane integrity [22-24]. However, numerous clinical studies and meta-analyses have confirmed this claim by demonstrating equal IOPlowering efficacy between PF and BAK-preserved prostaglandin analogs $[6,9,17]$.

\section{CONCLUSIONS}

Taken together, the current study demonstrated the noninferiority of PF latanoprost eye drops compared to BAKpreserved latanoprost eye drops in terms of IOP reduction, along with a good tolerability profile. The PF latanoprost $50 \mu \mathrm{g} / \mathrm{mL}$ formulation is a new multi-dose ophthalmic solution that may provide an efficacious alternative for glaucoma/ocular hypertension patients with existing ocular surface disease who do not tolerate eye drops with preservatives, especially when long-term treatment is required.

\section{ETHICS DECLARATIONS}

Ethical approval: The study protocol was prospectively approved by the National (Hellenic) Ethics Committee (NEC) (September 15, 2017) and the National (Hellenic) Organization for Medicines (EOF) (September 26, 2017). The study was conducted in compliance with the Declaration of Helsinki (2004) and Good Clinical Practice (GCP) guidelines. Relevant written informed consent was obtained from the patients prior to study enrollment. The study was registered in the EU Clinical Trials Register database with trial identification number 2017-00291029.

Conflict of interests: The study sponsor participated in the study design and interpretation of the data, the writing of the report, and the decision to submit the paper for publication. Theodosiadis P., Konstas A.G., Halkiadakis I., Dimera V., Koufakis D., Georgakopoulos K., and Kanonidou E. were investigators in the study, participated in the conduct of the study, and in the review and approval of the manuscript.

\section{FUNDING}

The study and medical writing support were sponsored by Pharmathen S.A., Greece. Kalantzi L., Margaritis A., and Soulele K. are employees of Pharmathen S.A. Theodosiadis P., Konstas A.G., Halkiadakis I., Dimera V., Koufakis D., Georgakopoulos CD., and Kanonidou E. are investigators in this study and have received financial compensation.

\section{ACKNOWLEDGEMENTS}

The authors wish to thank the investigators for allowing their patients to be included in this clinical study.

\section{REFERENCES}

1. SmPC. Xalatan 50 micrograms/mL Eye drops, solution Available from: https://www.medicines.org.uk/emc/product/1616/smpc.

2. Russo A, Riva I, Pizzolante T, Noto F, Quaranta L. Latanoprost ophthalmic solution in the treatment of open angle glaucoma or raised intraocular pressure: a review. Clin Ophthalmol. 2008;2(4):897-905. pmid: 19668444

3. Sharif NA, Kelly CR, Crider JY. Human trabecular meshwork cell responses induced by bimatoprost, travoprost, unoprostone, and other FP prostaglandin receptor agonist analogues. Invest Ophthalmol Vis Sci. 2003;44(2):715-21. doi: 10.1167/iovs.020323 pmid: 12556403 
4. Lim KS, Nau CB, O'Byrne MM, Hodge DO, Toris CB, McLaren $\mathrm{JW}$, et al. Mechanism of action of bimatoprost, latanoprost, and travoprost in healthy subjects. A crossover study. Ophthalmology. 2008;115(5):790-5 e4. doi: 10.1016/j.ophtha.2007.07.002 pmid: 18452763

5. Quaranta L, Gandolfo F, Turano R, Rovida F, Pizzolante T, Musig A, et al. Effects of topical hypotensive drugs on circadian IOP, blood pressure, and calculated diastolic ocular perfusion pressure in patients with glaucoma. Invest Ophthalmol Vis Sci. 2006;47(7):2917-23. doi: 10.1167/iovs.05-1253 pmid: 16799034 6. Rouland JF, Traverso CE, Stalmans I, Fekih LE, Delval L, Renault $D$, et al. Efficacy and safety of preservative-free latanoprost eyedrops, compared with BAK-preserved latanoprost in patients with ocular hypertension or glaucoma. $\mathrm{Br} \mathrm{J}$ Ophthalmol. 2013;97(2):196-200. doi: 10.1136/bjophthalmol-2012-302121 pmid: 23203707

7. Yang $Y$, Huang $C$, Lin $X, W u$, Ouyang $W$, Tang L, et al. $0.005 \%$ Preservative-Free Latanoprost Induces Dry Eye-Like Ocular Surface Damage via Promotion of Inflammation in Mice. Invest Ophthalmol Vis Sci. 2018;59(8):3375-84. doi: 10.1167/iovs.1824013 pmid: 30025085

8. Aptel F, Choudhry R, Stalmans I. Preservative-free versus preserved latanoprost eye drops in patients with open-angle glaucoma or ocular hypertension. Curr Med Res Opin. 2016;32(8):1457-63. doi: 10.1080/03007995.2016.1202818 pmid: 27310103

9. Aptel F, Pfeiffer N, Schmickler S, Clarke J, Lavin-Dapena C, Moreno-Montanes J, et al. Noninferiority of Preservative-free Versus BAK-preserved Latanoprost-timolol Fixed Combination Eye Drops in Patients With Open-angle Glaucoma or Ocular Hypertension. J Glaucoma. 2019;28(6):498-506. doi: 10.1097/IJG.0000000000001248 pmid: 31166287

10. Lopes NLV, Gracitelli CPB, Chalita MR, de Faria NVL. Ocular Surface Evaluation After the Substitution of Benzalkonium Chloride Preserved Prostaglandin Eye Drops by a Preservativefree Prostaglandin Analogue. Med Hypothesis Discov Innov Ophthalmol. 2019;8(1):52-6. pmid: 30923724

11. Steven DW, Alaghband P, Lim KS. Preservatives in glaucoma medication. $\mathrm{Br} J$ Ophthalmol. 2018;102(11):1497-503. doi: 10.1136/bjophthalmol-2017-311544 pmid: 29973365

12. SmPC. Monopost latanoprost $50 \mathrm{mcg} / \mathrm{ml}$ eye drops, solution in single-dose container. Available from: https://www.medicines.org.uk/emc/product/2978/smpc.

13. Stegemann S. Developing Drug Products in an Aging Society: From Concept to Prescribing. Sven (Ed.) ed: Springer International Publishing; 2016.

14. Bagnis A, Papadia M, Scotto R, Traverso CE. Antiglaucoma drugs: The role of preservative-free formulations. Saudi J Ophthalmol. 2011;25(4):389-94. doi: 10.1016/j.sjopt.2011.08.004 pmid: 23960953
15. Musch DC, Gillespie BW. The state of being noninferior. Ophthalmology. 2006;113(1):1-2. doi: 10.1016/j.ophtha.2005.09.015 pmid: 16389103

16. Gross RL, Sullivan EK, Wells DT, Mallick S, Landry TA, Bergamini MV. Pooled results of two randomized clinical trials comparing the efficacy and safety of travoprost $0.004 \% /$ timolol $0.5 \%$ in fixed combination versus concomitant travoprost $0.004 \%$ and timolol 0.5\%. Clin Ophthalmol. 2007;1(3):317-22. pmid: 19668487

17. Uusitalo H, Egorov E, Kaarniranta K, Astakhov $Y$, Ropo A. Benefits of switching from latanoprost to preservative-free tafluprost eye drops: a meta-analysis of two Phase IIIb clinical trials. Clin Ophthalmol. 2016;10:445-54. doi: 10.2147/OPTH.S91402 pmid: 27041987

18. Boimer C, Birt CM. Preservative exposure and surgical outcomes in glaucoma patients: The PESO study. J Glaucoma. 2013;22(9):730-5. doi: 10.1097/IJG.0b013e31825af67d pmid: 23524856

19. Munoz Negrete FJ, Lemij HG, Erb C. Switching to preservative-free latanoprost: impact on tolerability and patient satisfaction. Clin Ophthalmol. 2017;11:557-66. doi: 10.2147/OPTH.S126042 pmid: 28356710

20. Economou MA, Laukeland HK, Grabska-Liberek I, Rouland JF. Better tolerance of preservative-free latanoprost compared to preserved glaucoma eye drops: the 12-month real-life FREE study. Clin Ophthalmol. 2018;12:2399-407. doi: 10.2147/OPTH.S176605 pmid: 30538423

21. Cucherat M, Stalmans I, Rouland JF. Relative efficacy and safety of preservative-free latanoprost (T2345) for the treatment of open-angle glaucoma and ocular hypertension: an adjusted Indirect comparison meta-analysis of randomized clinical trials. J Glaucoma. 2014;23(1):e69-75. doi: 10.1097/IJG.0b013e3182a075e6 pmid: 23881267

22. Goody R, Woodley V, Henry S, Lawrence M, Olmiere C, editors. Intraocular pressure lowering effects of T2347 in normotensive nonhuman primates following once-daily topical administration. ARVO Annual Meeting; 2018 April 29-May 3, 2018; Honolulu, Hawaii.

23. McCarey B, Edelhauser $H$. In vivo corneal epithelial permeability following treatment with prostaglandin analogs [correction of analoges] with or without benzalkonium chloride. J Ocul Pharmacol Ther. 2007;23(5):445-51. doi: 10.1089/jop.2007.0024 pmid: 17941807

24. Baudouin C, Riancho L, Warnet JM, Brignole F. In vitro studies of antiglaucomatous prostaglandin analogues: travoprost with and without benzalkonium chloride and preserved latanoprost. Invest Ophthalmol Vis Sci. 2007;48(9):4123-8. doi: 10.1167/iovs.07-0266 pmid: 17724196 